\section{Vorbehandlung von Aktivkohle zur Bestimmung der Gruppenparameter AOS und AOX in Wasser}

W. Padowetz und H. Kästli

Zentrale Analytik der CIBA-GEIGY AG, CH-4002 Basel, Schweiz

\section{Treatment of actived carbon for the determination of the group parameters $\mathrm{AOS}$ and $\mathrm{AOX}$ in water}

In der Wasseranalytik gewinnen neben dem Gruppenparameter $\mathrm{AOCl}$ auch die Parameter $\mathrm{AOBr}, \mathrm{AOF}$ und $\mathrm{AOS}$ immer mehr an Bedeutung. Für die Bestimmung dieser Parameter sind Aktivkohlen efforderlich, die möglichst niedrige Blindwerte aufweisen. Die im Deutschen Einheitsverfahren zur Wasser-, Abwasser- und Schlammuntersuchung [1] empfohlene Aktivkohle F-400 weist niedrige Blindwerte für Chlor, Brom und Fluor auf und eignet sich daher für die Bestimmung dieser Parameter. Der Schwefelgehalt dieser Kohle beträgt jedoch durchschnittlich $9 \mathrm{mg} / \mathrm{g}$, was die Verwendung für die AOS-Bestimmung ausschließt. Aber auch andere käuflich erhältliche Aktivkohlen weisen Schwefelgehalte im Bereich von mehreren $\mathrm{mg} / \mathrm{g}$ auf. Es wurde daher vorgeschlagen, Aktivkohlen durch Verkokung von Glucose [2] herzustellen, und es wurde andererseits versucht, Aktivkohlen durch Oxidationsprozesse zu entschwefeln [3]. In der organischen Elementaranalyse wird die Reinigung des für die Sauerstoffbestimmung erforderlichen Gasrußes [4] durch Glühen im Wasserstoffstrom vorgenommen. Dieses Verfahren bewährt sich auch bei Aktivkohlen. Schwefelverbindungen werden dabei zu Schwefelwasserstoff bzw. zu elementarem Schwefel reduziert und mit dem Gasstrom entfernt. Aber auch der Halogengehalt kann auf diese Weise verringert werden, so daß es möglich ist, die Aktivkohle vor der Behandlung mit Wasserstoff mit starken Säuren (Salzsäure, Flußsäure) zu waschen, um den Aschegehalt zu senken.

\section{Experimentelles}

Apparatur. Rohrofen (W.C. Heraeus), Länge $320 \mathrm{~mm}$, regelbar bis $1000^{\circ} \mathrm{C}$.

Quarzrohr, Durchmesser $30 \mathrm{~mm}$, Länge $600 \mathrm{~mm}$, an einem Ende für Schlauchanschluß verjüngt.

Offprint requests to: W. Padowetz
Gasversorgung für Stickstoff und Wasserstoff mit Reduzierventilen und Flowmetern.

Arbeitsvorschrift. In das Quarzrohr einen Quarzwollepfropf einführen und darauf die Kohle so einfüllen, daß über der Kohle im Rohr ein Kanal verbleibt. Das Rohr in den Ofen einsetzen, die Verbindung zur Gasversorgung herstellen und das offene Rohrende mit einern durchbohrten Gummistopfen verschließen, mit dem ein Schlauch zum Ableiten der Gase verbunden ist. Zunächst nur Stickstoff zur Verdrängung der Luft durchleiten und den Ofen anheizen. Allmählich Wasserstoff zumischen und schließlich bei einer Temperatur von $950^{\circ} \mathrm{C}$ nur noch reinen Wasserstoff mit einer Strömungsgeschwindigkeit von 100 bis $150 \mathrm{ml} / \mathrm{min}$ durchleiten. Das Rohr regelmäBig zur Durchmischung der Kohle drehen. Der Verlauf der Reaktion kann durch Nachweis des Schwefelwasserstoffes im Gasstrom kontrolliert werden. Nach Beendigung wieder auf reinen Stickstoff umschalten und erkalten lassen.

Bei einem Ansatz von $15 \mathrm{~g}$ Aktivkohle (Fluka Artikel Nr. 5100) konnte durch diese Behandlung während $10 \mathrm{~h}$ der Schwefelgehalt von $7,5 \mathrm{mg} \mathrm{S} / \mathrm{g}$ auf $0,02 \mathrm{mg} \mathrm{S} / \mathrm{g}$ und der Chlorgehalt von $0,5 \mathrm{mg} \mathrm{Cl} / \mathrm{g}$ auf $0,01 \mathrm{mg} \mathrm{Cl} / \mathrm{g}$ gesenkt werden. Alle Werte wurden immer durch Verbrennung trockener Kohle erhalten.

Wegen der geringen Gehalte an Schwefel, Chlor und Asche eignet sich die Aktivkohle von E. Merck (Artikel 2186) besonders als Ausgangsmaterial für diese Behandlung und erreicht auch die nach AWWA Standard B 604 geforderte Jodzah1 [1]

\section{Literatur}

1. Deutsches Einheitsverfahren zur Wasser-, Abwasser- und Schlammuntersuchung: DIN 38409 T 14 (1985) Beuth Verlag, Berlin

2. Schnitzler M, Lévay W, Kuhn H, Sontheimer H (1983) Vom Wasser 61:263-276

3. Brandt $G$ (1987) Entwicklung einer Methode zur simultanen Bestimmung der Gruppenparameter $\mathrm{AOCl}, \mathrm{AOBr}$ und $\mathrm{AOS}$ als Beurteilungsgrößen für die Wasserbelastung. Dissertation, Universität GH-Paderborn

4. Ehrenberger F, Gorbach S (1973) Methoden der organischen Elementar- und Spurenanalyse. Verlag Chemie, Weinheim

Eingegangen am 16. März 1988

Fresenius Z Anal Chem (1988) 332:61-62 - (C) Springer Verlag 1988

\section{A simple method for the prediction of the GLC retention times of all the 209 PCB congeners}

\section{J. Devillers}

Institut Pasteur de Lyon, 77 rue Pasteur, F-69365 Lyon Cédex 7, France

\section{Einfaches Verfahren zur Voraussage der GLC-Retentionszeiten} aller 209 PCB-Congeneren

\section{Introduction}

The polychlorinated biphenyls (PCBs) are a group of xenobiotic chemicals that have attracted concern because of their worldwide distribution, their persistence in the environment, and their biological and human health effects. PCB molecules consist of a biphenyl nucleus carrying 1 to 10 chlorine atoms. Thus, there are 209 possible congeners ranging from the three monochlorobiphenyls to decachlorobiphenyl.

The physico-chemical and biological properties of these molecules only depend on the number and positions of chlorine atoms on the biphenyl nucleus.

Thus, the aim of the present study was to show that the highresolution capillary gas chromatographic behaviour of the 209 possible chlorinated biphenyls can be described by a simple coded numerical method of the chlorine substitution patterns.

\section{Experimental}

The experimental GLC retention times (RT) relative to the standard, octachloronaphthalene, for the 209 PCBs were taken from the literature [1]. 